\title{
A phenomenological three-parameter model for predicting the extent of outpatient palliative care
}

\author{
DIRK HARMS $^{1,2}$, MARK REINWALD ${ }^{1}$, FELISE KRAUTHAUSEN ${ }^{2}$, \\ DAGMAR STEIN $^{2}$ and PETER MARKUS DECKERT ${ }^{1,3}$
}

\author{
${ }^{1}$ Faculty of Medicine and Psychology, Brandenburg Medical School Theodor Fontane; ${ }^{2}$ Outpatient \\ Palliative Care Team Brandenburg; ${ }^{3}$ Faculty of Health Sciences Brandenburg, Brandenburg \\ Medical School Theodor Fontane, D-14770 Brandenburg an der Havel, Germany
}

Received November 11, 2020; Accepted June 1, 2021

DOI: $10.3892 / \mathrm{mco} .2021 .2356$

\begin{abstract}
Whether a patient receives general or specialized outpatient palliative cancer care rarely follows clear criteria, leading to undertreatment or overtreatment. Detailed scores exist to predict prognosis, but not treatment requirements, leaving caregivers to follow their intuition. As a phenomenological indicator incorporating possibly important subjective information, intuition may in fact be a helpful tool. In this prospective observational study, a score to estimate three global dimensions of patients' resources was applied: Medical prognosis, feeling of strength and feeling of support. The score results were correlated with the actual amount and effort of care required during the subsequent palliative care time. This phenomenological score correlated well with the performance index and the Hospice and Palliative care Evaluation score. Whilst various individual items correlated significantly with the score or its constituent parameters, there was no uniform coherent pattern, reflecting the complexity of palliative care and the potential value of this predictive tool.
\end{abstract}

\section{Introduction}

Both general practitioners and specialized palliative care teams tend to terminally ill cancer patients at home. Predicting the degree of care a patient needs, and hence, the suitable type or level of outpatient care required, is not trivial. Erring on either side will result in withholding adequate care, either by undertreating the index patient or by straining the resources available to other patients. A number of scales and scores in palliative care exists to estimate overall prognosis and partly

Correspondence to: Professor Peter Markus Deckert, Faculty of Medicine and Psychology, Brandenburg Medical School Theodor Fontane, Campus Brandenburg an der Havel, Hochstrasse 29,

D-14770 Brandenburg an der Havel, Germany

E-mail: deckert@klinikum-brandenburg.de

Key words: health care economics, medical ethics, health services, palliative care, health sciences, oncology to identify areas of need (1). The difference between general and specialized palliative care has often been defined by 'complexity', which is in itself an ill-defined term, and most often translates into the quantification of symptoms (2).

This analytic approach is largely driven by an effort to fragmentize the need for palliative care into manageable items, not least to justify costs. In its essence however, palliative care is a holistic approach, qualified by the need to keep in contact with the situation at large, whilst adequately addressing present symptoms (3). Hence, in this study, a tool to support decisionmaking in touch with the patient's situation and the caregivers' intuition was examined, attempting to establish criteria that are neither overly detailed nor arbitrary.

In particular with regard to palliative care, intuition appears to implicitly inform medical decisions more often than generally presumed. This happens rather as an unacknowledged subtext than a consciously employed and recorded method (4). Thus, to develop a scoring method, a phenomenological grounded theory approach was employed (5), evaluating existing literature on referral criteria and patient- or proxyreported outcome measures in palliative care (6-10). The results showed that three core aspects directed the perception of a terminally ill patient's multi-facetted situation.

In a first approach, these three items had been termed 'instability of the environment', 'weight of symptoms' and 'instability of the situation'. Upon validating this score, it underwent further development using the grounded theory process. The revised score then consisted of three dimensions termed 'perception of strength', 'perception of support and stability' and 'medical prognosis'. This score placed a clearer distinction between patient-reported and caregiver-observed criteria. In addition, it was easier to apply in practice, as instead of being deficiency-oriented, it was resource-oriented; that is, the higher the value, the better off the patient.

As these terms, such as a coordinate system, describe different dimensions, further consolidation into a single figure would come at the price of losing information gained in the process. Thus, it was decided to record the three dimensions separately in addition to combining them into one score.

The primary aim of this exploratory, non-interventional study was to assess whether this synthetic approach to summarizing the information gathered by the initial patient work-up 
in an ambulatory palliative care setting may predict the level of care required.

The secondary aims were to assess whether such an intuitive assessment and its documentation were feasible in daily practice.

\section{Patients and methods}

Study design and ethics approval. In a monocentric study, 142 patients referred for ambulatory palliative care were assessed in a mixed urban and rural area in Germany over a 2-year period between October 2015 and September 2017. Inclusion criteria were advanced and progredient incurable disease with a short life expectancy of weeks to months and complex symptoms. Exclusion criteria were incapability to give informed consent or lack thereof, and incapability to participate in a complete initial anamnestic and physical assessment. All patients referred to ambulatory palliative care within the study period were screened and finally included into the study. The present study was approved by the Ethics Committee of the Medizinische Hochschule Brandenburg Theodor Fontane and conducted according to the Declaration of Helsinki as adopted by the World Medical Organization in October 2013 (11), and all patients provided written informed consent to participate.

Patient characteristics. The distribution of the sexes of the total study population was skewed towards males $(n=83$; $58 \%$ vs. $n=59 ; 42 \%$ ). Excluding one pediatric patient from further evaluation, the median age was 73 years (age range, 42-91 years), and $18 \%$ were living alone. In the vast majority of patients, the life-limiting diagnosis was malignant disease (90.1\%). Amongst the 60 patients evaluated using the final version of the scoring system, the distribution of sexes was slightly more even with 33 male and 27 female patients (55 and 45\%, respectively), the median age was 76 years (age range, 50-91 years), and $88.3 \%$ of the patients had a malignant disease as the primary diagnosis; Table I shows the demographic characteristics, Table II the medical characteristics of the patients.

Study procedure. The assessments were performed by one of three participating palliative care physicians, who contributed 67,28 , and $5 \%$ of the assessments for this study. Patients consent only covered assessment by their assigned primary palliative care physician. All participating physicians were board certified in palliative medicine with $>20$ years professional experience each. The initial outpatient palliative care visit routinely consisted of recording a multi-dimensional patient history including medical, family and psychosocial aspects, their current situation and patients' perceptions and expectations, followed by a physical examination and subsequent care-planning for the immediate future. Upon completion of this visit, the physician rated and documented the score parameters. After the end of ambulatory palliative care, items describing each patient's pre-existing condition and the palliative care effort delivered (documentation items) were collected in a pseudonymized database.

Development and evaluation of the score. A total of 82 patients were assessed with the first scoring approach described in the introduction. Upon analyzing the results, both as to practical applicability and prognostic value for the required extent of palliative care, it was revisited, and for the subsequent 60 patients, the score comprising the parameters 'perception of strength', 'perception of support and stability', and 'medical prognosis' was used. Upon performing the initial patient workup, the physician gave an estimate of these parameters using a positive visual analog scale, where 10 was the best possible or 'healthy' realization of each parameter and 0 was the worst possible realization. The complete score for each patient was calculated as the sum of the three constituent parameters, and thus had a range of 0-30.

Both the complete score and its constituent parameters were compared with two established scores, the Hospice and Palliative care Evaluation (HOPE) (9) and the Karnofsky index (KI) (12), and with documentation items indicating the actual care effort delivered to a patient. The established scores were applied by the same physician at the same time as the experimental score.

Statistical analysis. Statistical calculations were performed using SPSS version 21.0 (TBM Corp). Numerically scaled values of the individual observation items as listed in Figs. 1 and 2 were compared to the total score value using a Pearson's correlation analysis, followed by subsequent multivariate regression analysis, where correlations $>0.2$ were found to be significant. As all items were at least interval scaled, this analysis was deemed appropriate to maintain a uniform evaluation method. For the initial version of the score, a sample size of $n=83$, and for the final version, a sample size of $n=60$ was evaluated. For the sample size and distribution, the indicated Pearson's $r$ correlation coefficients were associated with the following levels of significance according to the two-sided Student's t-test: $\mathrm{r}>0.3, \mathrm{P}<0.01 ; \mathrm{r}>0.4, \mathrm{P}<0.001$; $\mathrm{r}>0.5, \mathrm{P}<0.0001$. Correlation coefficients reaching a level of significance of $\mathrm{P}<0.01$ have been highlighted in bold italics in the figures.

\section{Results}

Inter-rater variability. A direct comparison between raters on the same patient was methodologically impossible. Comparing the two physicians who together provided $>90$ percent of the assessments, descriptive statistics revealed mean rating scores of 4.0/10 vs. 6.10/10 (perception of support and stability), $7.5 / 10$ vs. $8.0 / 10$ (perception of strength) and $7.95 / 10$ vs. $7.5 / 10$ (medical condition), respectively. Due to the small number of interviews, a meaningful comparison with the third participating physician was not possible.

Primary score. To provide an impression of the actual score figures with the initial scoring approach, the means (and confidence intervals) were 19.67 (12.75-26.59) for the total and 5.13 (0.87-9.39) for 'instability of the environment', 7.28 (4.07-10.50) for 'weight of symptoms' and 7.25 (4.12-10.39) for 'instability of the situation'. Here, the total score correlated well with all three constituent parameters, but significant correlations were found only of the total score, "weight of symptoms' and 'instability of the situation' as score parameters, and the documentation items 'number of medical visits', 
Table I. Demographic patient characteristics.

\begin{tabular}{lccc}
\hline Characteristic & Female & Male & Total \\
\hline Sex, \% & 41.5 & 58.5 & 100 \\
Age, years, mean & 73.5 & 71.7 & 72.4 \\
Age, years, median & 72 & 75 & 73 \\
Age, $95 \%$ confidence interval & $53.1-93.9$ & $50.3-91.6$ & $51.7-92.5$
\end{tabular}

Table II. Medical patient characteristics.

\begin{tabular}{|c|c|c|}
\hline Diagnostic category & $\%$ & $\mathrm{n}$ \\
\hline Total & 100 & 142 \\
\hline Malignant & 90.1 & 128 \\
\hline Hematologic & 9.2 & 13 \\
\hline Solid, intermediate stage & 17.6 & 25 \\
\hline Solid, late stage & 63.4 & 90 \\
\hline Non-malignant & 6.3 & 9 \\
\hline Unknown & 3.5 & 5 \\
\hline \multicolumn{3}{|l|}{ Malignant diagnoses } \\
\hline Leukemia & 2.1 & 3 \\
\hline Lymphoma & 7 & 10 \\
\hline Central nervous system & 1.4 & 2 \\
\hline Head and neck & 5.6 & 8 \\
\hline Esophageal & 2.1 & 3 \\
\hline Gastric & 4.2 & 6 \\
\hline Intestinal & 1.4 & 2 \\
\hline Colorectal & 7 & 10 \\
\hline Liver & 7 & 10 \\
\hline Pancreatic & 6.3 & 9 \\
\hline Lung and pleura & 16.9 & 24 \\
\hline Breast & 6.3 & 9 \\
\hline Gynacological & 2.1 & 3 \\
\hline Prostate & 5.6 & 8 \\
\hline Urogenital & 7 & 10 \\
\hline Melanoma, sarcoma or neuroendocrine & 2.8 & 4 \\
\hline Carcinoma of unknown primary & 4.9 & 7 \\
\hline
\end{tabular}

'number of urgent medical visits' and 'number of medical phone calls'. None of these reached a correlation coefficient of 0.3, which was considered the significance threshold; thus, this score was not pursued any further.-

Revised score. The means (and confidence intervals) of the revised score were 16.1 (7.3-24.9) for the total score, 4.6 (0-9.5) for 'perception of strength', 6.8 (3.2-10.4) for 'perception of support and stability' and 4.7 (0.1-9.3) for 'medical prognosis'. From sample size and distribution, the indicated Pearson's $r$ correlation coefficients were associated with the following significance levels: $r>0.3, \mathrm{P}<0.01 ; \mathrm{r}>0.4$, $\mathrm{P}<0.001$; and $\mathrm{r}>0.5, \mathrm{P}<0.0001$. These results are summarized in Figs. 1 and 2, where statistically significant correlations $(\mathrm{P}<0.01)$ have been highlighted.
With correlation coefficients of 0.66 and 0.49 , the scores of the present study correlated well with KI and HOPE scores, respectively; the latter correlation being inverse as HOPE increases with symptom load, whereas $\mathrm{KI}$ and the experimental score increase with performance and resources. The constituent dimensions 'perception of strength' and 'medical prognosis' (correlation coefficients 0.79 and 0.78 , respectively) most strongly predicted the total score (Figs. 1 and 2).

The actual care effort delivered to the patient did not correlate with rural/urban residence, age, sex, number of symptom-relieving drugs taken, assigned degree of nursing care, tumor stage or number of residents in the household.

Looking at individual documentation items indicating the palliative care efforts actually delivered, none correlated 


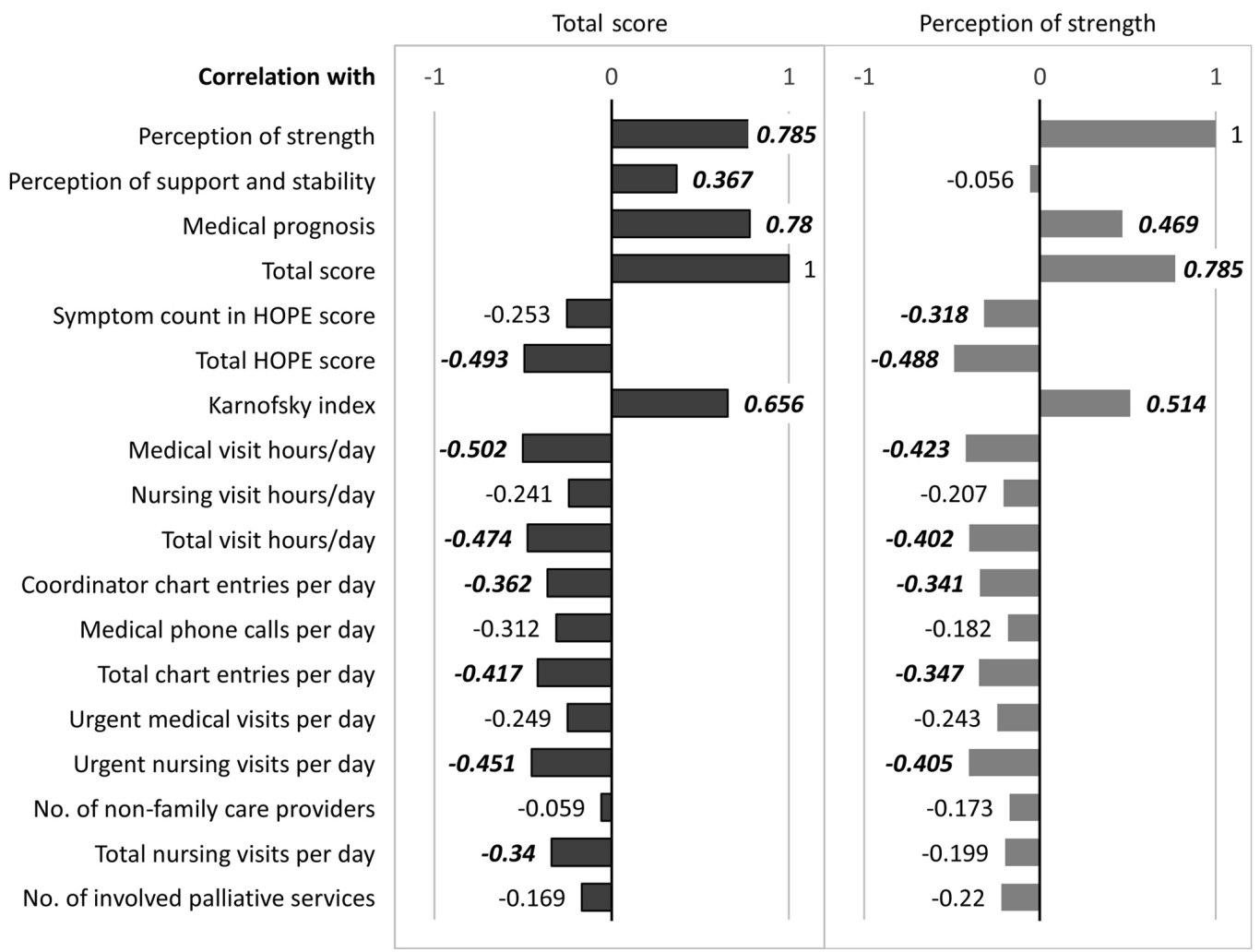

Figure 1. Correlation of palliative care documentation items with the complete score and the score parameters 'perception of strength'. Significant correlations are printed in bold italics. Numerical values of the individual observation items listed were compared to the total score value using Pearson's correlation analysis for linear correlation. As all items were at least interval scaled, this calculation was deemed appropriate to maintain a uniform evaluation method. HOPE, Hospice and Palliative care Evaluation.

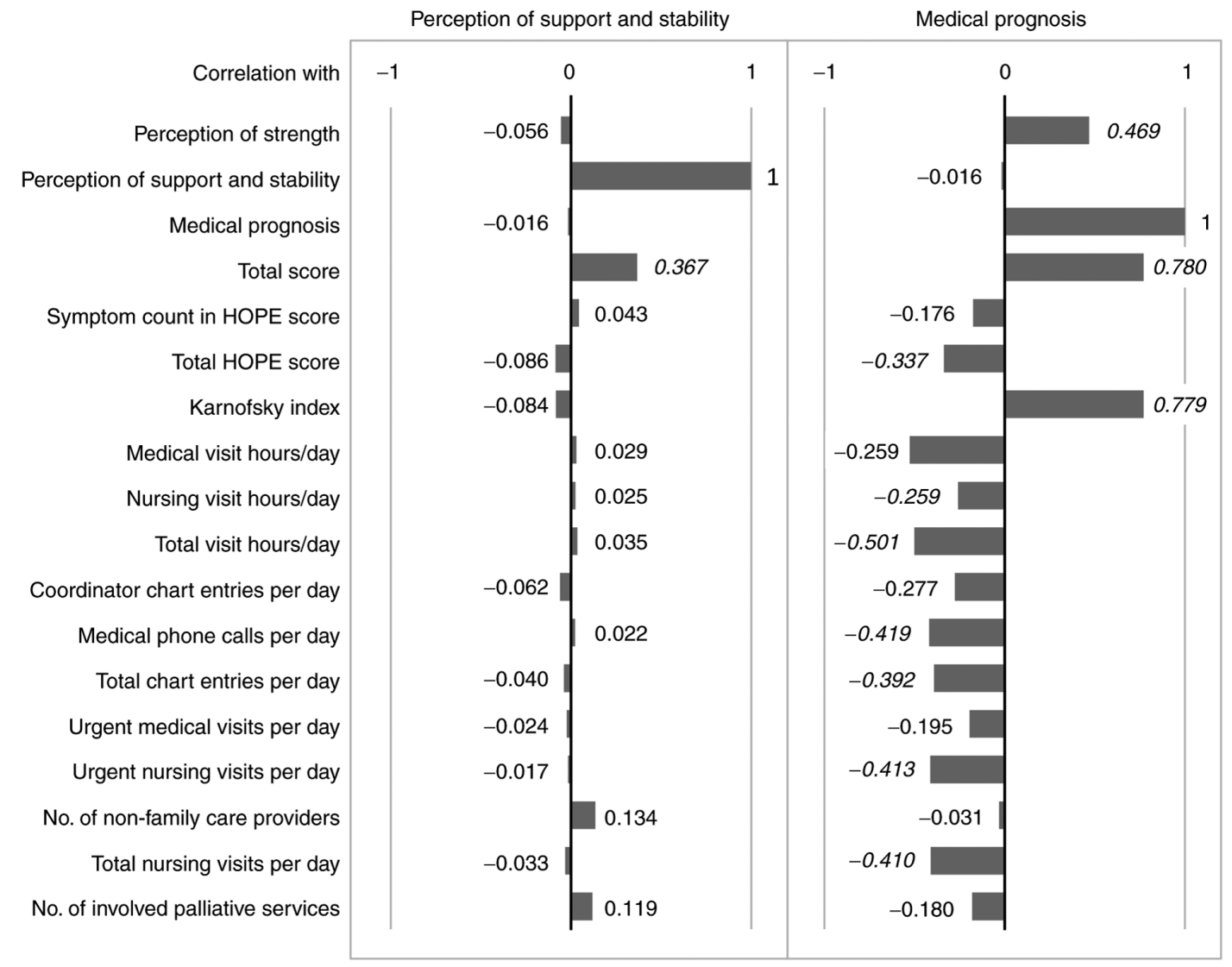

Figure 2. Correlation of palliative care documentation items with the score parameters 'perception of support and stability' and 'medical prognosis'. Significant correlations are printed in italics. 
with 'perception of support and stability' (Fig. 2). A subgroup defined by this parameter in which the score was $<5$ however, showed consistently high documentation item values for care effort, consistent with high care requirements due to little existing support (data not shown).

Both the total score and its constituent parameters 'perception of strength' and 'medical prognosis' correlated inversely with the following documentation items for palliative care effort: Time for medical visits, time for medical and nursing visits, number of entries by coordinator and by palliative physician, and number of urgent nursing visits. The number of medical phone calls and the total number of nursing visits per day correlated inversely with 'medical prognosis' and with the total score.

\section{Discussion}

There are several different scoring systems available for evaluating palliative care, starting with the KI and ECOG scores developed for use in oncology. These are still being amended to develop newer more accurate models $(1,9,13)$. However, the focus of these models is primarily on symptom severity and overall prognosis, rather than estimation of resources needed $(1,6,14,15)$. In 2016 , an international group found six predominant themes, which largely corresponded to the dimensions of the score developed in the present study (10). Far from a one-to-one translation, other groups also came across similar dimensions to evaluate the need for palliative care. An international consensus paper on referral criteria for outpatient specialty palliative care by Hui et al (16) comprised 11 major and 36 minor criteria. Thus, a demand for brief, patient-oriented assessment tools has been recognized and is being addressed by newer scales, such as the integrated palliative care outcome scale (IPOS) $(13,15)$.

In the present study, the practical reliability of intuitive decisions regarding resource allocations in palliative care, and whether this approach can be systematically incorporated into structured decision-making was assessed. By applying a phenomenological method to the experience of the examining physician $(4,5)$, it was assumed that intuition facilitates perceptions that allow for a more focused comprehensive picture, in that it does not seek separated parts, but incorporates several meaningful entities which are difficult to measure. The perceived entities arise and differ depending on the question asked. Hence, the three dimensions of the score employed here feed back on the reported perceptions of the rating physician and focuses their attention towards them.

The score introduced here strongly correlated with documentation items indicating palliative care effort, such as the number of interactions of caregivers, the time required and the number of emergency visits. In this, it may actually be suitable to support decision-making about general or specialized palliative care assignments. Yet the present study has clear limitations. The numbers of patients as well as rating physicians were small, and it was a single center study. If one were to 'calibrate' this score, a larger population or a less diverse setting as to the patients' environmental situation would be required. In addition, it would be interesting to apply it in different countries and cultural settings to see to what extent its questions and the perceptions informing their answers are culturally defined or universal.

A very stimulating result in this context is the lack of correlation between the initial 'perception of support and stability' and the outcomes of interest. One explanation could be a principal inaccuracy of assessment due to rapid changes in the event of massive disturbances leading to hospital admissions or relocation to the hospice. Therefore, this constituent parameter of the score may be particularly prone to culturally defined perceptions, and further phenomenological research should deal with an intercultural and situational meaning of support. Another explanation could be that this estimator serves as an internal correction to the others. This is, in part, supported by the observation that there may be a threshold effect where patients with 'less than sufficient' perception of support and stability require a greater degree of palliative care. To see this statistically would again require larger numbers, but a closer prospective look at this parameter in individual future patients may help to resolve this question.

The good correlation of the scoring system developed in the present study with that of the KI for general performance poses the question whether both measure the same quality. The KI basically pertains to general physical fitness, not to dimensions of prognosis and self-perception of the patient. Still, these qualities are associated with each other; improved physical performance is likely associated with an improved perception of strength and less subjective need of being cared for.

To test for this, the KI was statistically incorporated into the scoring system, either as an additional or as a substitute for the apparently weak predictor 'perception of support and stability'. In both cases, it performed worse in predicting the actual care effort than the original score did (data not shown), supporting a preliminary view that both do not measure the same. Again, to confirm this statistically rather than narratively will require larger datasets.

Beyond that however, the value of the present approach by its intention should not be limited to an improved prediction of care effort, but support a more comprehensive attitude of interacting with the patient, their environment and a patientcentered medical view, and to do so without being inferior in prediction to existing scores.

The idea of this approach is to counter a tendency towards ever more detailed itemizations and to direct time and attention back to inter-personal patient attention and care. In this, it is a radically pragmatic approach to utilize caregiver's capabilities of intuitive assessment in an explicit and reflected manner. It must be emphasized however, that this does not in any way imply to save time by placing a precedence of 'gut feeling' over factual assessment. To the opposite, the score was gathered only after a thorough initial patient workup.

Whilst this score may help to estimate the amount and intensity of palliative care required, a question not addressed here is its cutoff. Obviously, no precise threshold, but only scale areas indicating 'rather general palliative care', 'rather specialized palliative care' or 'indeterminate' may possibly be established in the future.

In summary, the present study explored a 3D phenomenological model and suggests, without yet defining a practically applicable discriminator, that their combination correlates 
with the required extent of care at least as well as existing scores. It was also suggested that performing and documenting this score is feasible in the actual everyday work of ambulatory palliative care. To validate the system for prospective application and decision-making; however, will require additional, larger and multi-center studies.

\section{Acknowledgements}

Not applicable.

\section{Funding}

The study was in part funded by internal institutional funds from the Brandenburg Medical School Theodor Fontane.

\section{Availability of data and materials}

The datasets used and/or analyzed during the present study are available from the corresponding author on reasonable request.

\section{Authors' contributions}

DH, MR and PMD conceived the study, and analyzed and interpreted the data. DH performed the background research. DH, DS and FK collected the data. PMD wrote the manuscript. DH, MR and PMD edited the manuscript. DH and PMD confirm the authenticity of all the raw data. All authors have read and approved the final manuscript.

\section{Ethics approval and consent to participate}

The present study was approved by the Ethics Committee of the Brandenburg Medical School Theodor Fontane (approval no. E-01-20180716) and was performed in accordance with the Declaration of Helsinki. Consent was obtained as described in the Methods section. All patients provided written informed consent to participate.

\section{Patient consent for publication}

Not applicable.

\section{Competing interests}

The authors declare that they have no competing interests.

\section{References}

1. Bausewein C, Le Grice C, Simon S and Higginson I; PRISMA The use of two common palliative outcome measures in clinical care and research: A systematic review of POS and STAS. Palliat Med 25: 304-313, 2011.
2. Kamps H and Harms D: Komplexe hausarztmedizin. Z Allg Med 87: 5, 2011.

3. Julião M, Sobral MA,Calcada P, Antunes B, Nunes B, Braganca A Runa D, Faria de Sousa P, Chochinov HM and Bruera E: 'Truly holistic?' Differences in documenting physical and psychosocial needs and hope in Portuguese palliative patients. Palliat Support Care 19: 69-74, 2021.

4. Todd PM and Gigerenzer G: Precis of Simple heuristics that make us smart. Behav Brain Sci 23: 727-780, 2000.

5. Giorgi A: An affirmation of the phenomenological psychological descriptive method: A response to Rennie (2012). Psychol Methods 19: 542-551, 2014

6. Glare P, Shariff I and Thaler HT: External validation of the number of risk factors score in a palliative care outpatient clinic at a comprehensive cancer center. J Palliat Med 17: 797-802, 2014.

7. Maltoni M, Scarpi E, Pittureri C, Martini F, Montanari L, Amaducci E, Derni S, Fabbri L, Rosati M, Amadori D and Nanni O: Prospective comparison of prognostic scores in palliative care cancer populations. Oncologist 17: 446-454, 2012.

8. Shinall MC, Ely EW, Karlekar M, Robbins SG, Chandrasekhar R and Martin SF: Psychometric properties of the FACIT-Pal 14 administered in an outpatient palliative care clinic. Am J Hosp Palliat Care 35: 1292-1294, 2018

9. Stiel S, Pollok A, Elsner F, Lindena G, Ostgathe C, Nauck F and Radbruch L: Validation of the symptom and problem checklist of the german hospice and palliative care evaluation (HOPE). J Pain Symptom Manage 43: 593-605, 2012.

10. Hui D, Meng YC, Bruera S, Geng Y, Hutchins R, Mori M, Strasser F and Bruera E: Referral criteria for outpatient palliative cancer care: A systematic review. Oncologist 21: 895-901, 2016.

11. World Medical Association: World Medical Association Declaration of Helsinki: Ethical principles for medical research involving human subjects. JAMA 310: 2191-2194, 2013.

12. Crooks V, Waller S, Smith T and Hahn TJ: The use of the karnofsky performance scale in determining outcomes and risk in geriatric outpatients. J Gerontol 46: M139-M144, 1991.

13. Roch C, Palzer J, Zetzl T, Stork S, Frantz S and van Oorschot B: Utility of the integrated palliative care outcome scale (IPOS): A cross-sectional study in hospitalised patients with heart failure. Eur J Cardiovasc Nurs 19: 702-710, 2020.

14. Etkind SN,LovellN, Bone AE, Guo P,Nicholson C, Murtagh FEM and Higginson IJ: The stability of care preferences following acute illness: A mixed methods prospective cohort study of frail older people. BMC Geriatr 20: 370, 2020.

15. Murtagh FE, Ramsenthaler C, Firth A, Groeneveld EI, Lovell N, Simon ST, Denzel J, Guo P, Bernhardt F, Schildmann E, et al: A brief, patient- and proxy-reported outcome measure in advanced illness: Validity, reliability and responsiveness of the Integrated Palliative care Outcome Scale (IPOS). Palliat Med 33: 1045-1057, 2019.

16. Hui D, Mori M, Watanabe SM, Caraceni A, Strasser F, Saarto T, Cherny N, Glare P, Kaasa S and Bruera E: Referral criteria for outpatient specialty palliative cancer care: An international consensus. Lancet Oncol 17: e552-e559, 2016. 